\title{
Numerical Simulation for the Fractional SIRC Model and Influenza A
}

\author{
M. M. Khader ${ }^{1,2}$, N. H. Sweilam ${ }^{3, *}$, A. M. S. Mahdy ${ }^{4}$ and N. K. Abdel Moniem ${ }^{5}$ \\ ${ }^{1}$ Department of Mathematics and Statistics, College of Science, Al-Imam Mohammad Ibn Saud Islamic University (IMSIU), Riyadh: \\ 11566, Saudi Arabia \\ ${ }^{2}$ Department of Mathematics, Faculty of Science, Benha University, Benha, Egypt \\ ${ }^{3}$ Department of Mathematics, Faculty of Science, Cairo University, Giza, Cairo, Egypt \\ ${ }^{4}$ Department of Mathematics, Faculty of Science, Zagazig University, Zagazig, Egypt \\ ${ }^{5}$ Information Technology Department, National Cancer Institute, Giza, Cairo, Egypt
}

Received: 13 May. 2013, Revised: 17 Sep. 2013, Accepted: 18 Sep. 2013

Published online: 1 May. 2014

\begin{abstract}
In this paper, A Chebyshev spectral method is presented to study the deals with the fractional SIRC model associated with the evolution of influenza A disease in human population. The properties of the Chebyshev polynomials are used to derive an approximate formula of the Caputo fractional derivative. This formula reduces the SIRC model to the solution of a system of algebraic equations which is solved using Newton iteration method. The convergence analysis and an upper bound of the error of the derived formula are given. We compared our numerical solutions with those numerical solutions using fourth-order Runge-Kutta method. The obtained results of the SIRC model show the simplicity and the efficiency of the proposed method. Also, illustration for propagation of influenza A virus and the relation between the four cases of it along the time at the fractional derivative are given.
\end{abstract}

Keywords: SIRC model; Caputo fractional derivative; Chebyshev spectral method; convergence analysis; fourth-order Runge-Kutta method.

\section{Introduction}

It is well known that the fractional differential equations (FDEs) have been the focus of many studies due to their frequent appearance in various applications such as in fluid mechanics, viscoelasticity, biology, physics and engineering applications, for more details see for example ([18], [20]). Consequently, considerable attention has been given to the efficient numerical solutions of the FDEs of physical interest, because it is difficult to find the exact solutions for it. Different numerical methods have been proposed in the literature for solving the FDEs, see ([9]-[17], [23]-[29]).

Mathematical models have become important tools in analyzing the spread and control of infectious diseases. Under-standing the transmission characteristics of infectious diseases in communities, regions, and countries can lead to better approaches to decrease the transmission of these diseases [7].

Influenza is transmitted by a virus that can be of three different types, namely $A, B$, and $C$ [19]. Among these, the virus $A$ is epidemiologically the most important one for human beings, because it can recombine its genes with those of strains circulating in animal populations, such as birds, swine, horses, and so forth ([1], [30]). Over the last two decades, a number of epidemic models for predicting the spread of influenza through human population have been proposed based on either the classical susceptible-infected-removed $(S I R)$ model developed by Kermack and McKendrick [8].

In this paper, we use the collocation spectral method to study the behavior of the approximate solution of the following fractional model of SIRC

$$
\begin{aligned}
& D^{\alpha} S(t)=\mu(1-S)-\beta S I+\gamma C, \\
& D^{\alpha} I(t)=\beta S I+\sigma \beta C I-(\mu+\theta) I, \\
& D^{\alpha} R(t)=(1-\sigma) \beta C I+\theta I-(\mu+\delta) R, \\
& D^{\alpha} C(t)=\delta R-\beta C I-(\mu+\gamma) C,
\end{aligned}
$$

with the following initial conditions

$$
S(0)=s_{0}, \quad I(0)=i_{0}, \quad R(0)=r_{0}, \quad C(0)=c_{0} .
$$

\footnotetext{
* Corresponding author e-mail: nsweilam@ @sci.cu.edu.eg
} 
Where $D^{\alpha}$ is the Caputo fractional derivative, with respect to time $t$. In which $S=S(t), I=I(t), R=R(t)$ and $C=C(t)$ represent the proportions of susceptible, infectious, recovered and cross-immune, respectively. The model assumes a population of constant size, $N$, so that $N=S+I+R+C$, where provides an interpretation of the model parameters, $\mu=0.01 y^{-1}$ is the mortality rate, $\theta=365 / 3 y^{-1}$ is the rate of progression from infective to recovered per year, $\delta=0.625 y^{-1}$ is rate of progression from recovered to cross-immune per year, $\gamma=0.35 y^{-1}$ is rate of progression from recovered to susceptible per year, $\sigma$ is the recruitment rate of cross-immune into the infective $(0 \leq \sigma \leq 1)$ and $\beta=1200$ is the contact rate per year. Because model (1) monitors the dynamics of human populations, all the parameters are assumed to be nonnegative. Furthermore, it can be shown that all state variables of the model are nonnegative for all time $t \geq 0$ ([2], [3], [21]).

Further details on the biological motivation and the associated assumptions are given in ([4]-[6]).

Note that, when $\alpha=1$, we get the standard form of this system as follows

$$
\begin{aligned}
& \frac{d S}{d t}=\mu(1-S)-\beta S I+\gamma C, \\
& \frac{d I}{d t}=\beta S I+\sigma \beta C I-(\mu+\theta) I, \\
& \frac{d R}{d t}=(1-\sigma) \beta C I+\theta I-(\mu+\delta) R, \\
& \frac{d C}{d t}=\delta R-\beta C I-(\mu+\gamma) C,
\end{aligned}
$$

where the parameter $\beta$ is the contact rate for the influenza disease also called the rate of transmission for susceptible to infected, $\gamma^{-1}$ is the cross-immune period, $\theta^{-1}$ is the infectious period, $\delta^{-1}$ is the total immune period and $\sigma$ is the fraction of the exposed cross-immune individuals who are recruited in an unit time into the infective subpopulation ([2], [6]).

In this section, we introduce some necessary definitions and mathematical preliminaries of the fractional calculus theory that will be required in the present paper.

\section{Definition 1.}

The Caputo fractional derivative operator $D^{\alpha}$ of order $\alpha$ is defined in the following form

$D^{\alpha} f(x)=\frac{1}{\Gamma(m-\alpha)} \int_{0}^{x} \frac{f^{(m)}(t)}{(x-t)^{\alpha-m+1}} d t, \quad \alpha>0, \quad x>0$, where $m-1<\alpha \leq m, m \in \mathbb{N}$.

Similar to integer-order differentiation, Caputo fractional derivative operator is a linear operation

$$
D^{\alpha}(\lambda f(x)+\mu g(x))=\lambda D^{\alpha} f(x)+\mu D^{\alpha} g(x),
$$

where $\lambda$ and $\mu$ are constants. For the Caputo's derivative we have [20]

$$
D^{\alpha} C=0, \quad C \text { is a constant, }
$$

$D^{\alpha} x^{n}= \begin{cases}0, & \text { for } \quad n \in \mathbb{N}_{0} \text { and } n<\lceil\alpha\rceil \\ \frac{\Gamma(n+1)}{\Gamma(n+1-\alpha)} x^{n-\alpha}, & \text { for } \quad n \in \mathbb{N}_{0} \text { and } n \geq\lceil\alpha\rceil\end{cases}$

We use the ceiling function $\lceil\alpha\rceil$ to denote the smallest integer greater than or equal to $\alpha$ and $\mathbb{N}_{0}=\{0,1,2, \ldots\}$. Recall that for $\alpha \in \mathbb{N}$, the Caputo differential operator coincides with the usual differential operator of integer order. For more details on fractional derivatives, definitions and its properties see ([18], [20]).

The organization of this paper is as follows. In the next section, we derive an approximate formula for fractional derivatives using Chebyshev series expansion. In section 3, we study the error analysis of the introduced approximate formula. In section 4 , we give the procedure of the solution using Chebyshev collocation method. In section 5, we present the numerical implementation of the proposed technique. The conclusion is given in section 6 .

\section{Derivation of the approximate formula}

The well known Chebyshev polynomials [22] are defined on the interval $[-1,1]$ and can be determined with the aid of the following recurrence formula

$T_{n+1}(z)=2 z T_{n}(z)-T_{n-1}(z), T_{0}(z)=1, T_{1}(z)=z, n=$ $1,2, \ldots$. The analytic form of the Chebyshev polynomials $T_{n}(z)$ of degree $n$ is given by

$T_{n}(z)=n \sum_{i=0}^{\left[\frac{n}{2}\right]}(-1)^{i} 2^{n-2 i-1} \frac{(n-i-1) !}{(i) !(n-2 i) !} z^{n-2 i}, n=2,3, \ldots$,

where $[n / 2]$ denotes the integer part of $n / 2$. The orthogonality condition is

$$
\int_{-1}^{1} \frac{T_{i}(z) T_{j}(z)}{\sqrt{1-z^{2}}} d z= \begin{cases}\pi, & \text { for } i=j=0 \\ \frac{\pi}{2}, & \text { for } i=j \neq 0 \\ 0, & \text { for } i \neq j\end{cases}
$$

In order to use these polynomials on the interval $[0,1]$ we define the so called shifted Chebyshev polynomials by introducing the change of variable $z=2 t-1$. The shifted Chebyshev polynomials are defined as

$$
T_{n}^{*}(t)=T_{n}(2 t-1)=T_{2 n}(\sqrt{t})
$$

The analytic form of the shifted Chebyshev polynomials $T_{n}^{*}(t)$ of degree $n$ is given by

$$
T_{n}^{*}(t)=n \sum_{k=0}^{n}(-1)^{n-k} \frac{2^{2 k}(n+k-1) !}{(2 k) !(n-k) !} t^{k}, \quad n=2,3, \ldots
$$

The function $x(t)$, which belongs to the space of square integrable functions in $[0,1]$, may be expressed in terms of shifted Chebyshev polynomials as

$$
x(t)=\sum_{i=0}^{\infty} c_{i} T_{i}^{*}(t)
$$


where the coefficients $c_{i}$ are given by

$c_{i}=\frac{2}{\pi h_{i}} \int_{0}^{1} \frac{x(t) T_{i}^{*}(t)}{\sqrt{t-t^{2}}} d t, \quad h_{0}=2, \quad h_{i}=1, \quad i=1,2, \ldots$

In practice, only the first $(m+1)$-terms of shifted Chebyshev polynomials are considered. Then we have

$$
x_{m}(t)=\sum_{i=0}^{m} c_{i} T_{i}^{*}(t)
$$

The main approximate formula of the fractional derivative of $x_{m}(t)$ is given in the following theorem.

\section{Theorem 1.}

Let $x(t)$ be approximated by Chebyshev polynomials as in (10) and also suppose $\alpha>0$, then

$$
D^{\alpha}\left(x_{m}(t)\right)=\sum_{i=\lceil\alpha\rceil}^{m} \sum_{k=\lceil\alpha\rceil}^{i} c_{i} w_{i, k}^{(\alpha)} t^{k-\alpha},
$$

where $w_{i, k}^{(\alpha)}$ is given by

$$
w_{i, k}^{(\alpha)}=(-1)^{i-k} \frac{2^{2 k} i(i+k-1) ! \Gamma(k+1)}{(i-k) !(2 k) ! \Gamma(k+1-\alpha)} .
$$

Proof.Since the Caputo's fractional differentiation is a linear operation we have

$$
D^{\alpha}\left(x_{m}(t)\right)=\sum_{i=0}^{m} c_{i} D^{\alpha}\left(T_{i}^{*}(t)\right)
$$

Employing Eqs.(4) and (5) in Eq.(7), we obtain

$$
D^{\alpha} T_{i}^{*}(t)=0, \quad i=0,1, \ldots,\lceil\alpha\rceil-1, \quad \alpha>0 .
$$

Therefore, for $i=\lceil\alpha\rceil,\lceil\alpha\rceil+1, \ldots, m$, and by using Eqs.(4) and (5) in Eq.(7), we get

$$
\begin{aligned}
D^{\alpha} T_{i}^{*}(t) & =i \sum_{k=\lceil\alpha\rceil}^{i}(-1)^{i-k} \frac{2^{2 k}(i+k-1) !}{(i-k) !(2 k) !} D^{\alpha} t^{k} \\
& =i \sum_{k=\lceil\alpha\rceil}^{i}(-1)^{i-k} \frac{2^{2 k}(i+k-1) ! \Gamma(k+1)}{(i-k) !(2 k) ! \Gamma(k+1-\alpha)} t^{k-\alpha}
\end{aligned}
$$

A combination of Eqs.(13), (14) and (15) leads to the desired result and completes the proof of the theorem.

\section{Error analysis}

In this section, special attention is given to study the convergence analysis and evaluate an upper bound of the error of the proposed formula.

Theorem 2. (Chebyshev truncation theorem) [22]
The error in approximating $x(t)$ by the sum of its first $m$ terms is bounded by the sum of the absolute values of all the neglected coefficients. If

$$
x_{m}(t)=\sum_{k=0}^{m} c_{k} T_{k}(t)
$$

then

$$
E_{T}(m) \equiv\left|x(t)-x_{m}(t)\right| \leq \sum_{k=m+1}^{\infty}\left|c_{k}\right|,
$$

for all $x(t)$, all $m$, and all $t \in[-1,1]$.

Theorem 3. [16]

The Caputo fractional derivative of order $\alpha$ for the shifted Chebyshev polynomials can be expressed in terms of the shifted Chebyshev polynomials themselves in the following form

$$
D^{\alpha}\left(T_{i}^{*}(t)\right)=\sum_{k=\lceil\alpha\rceil}^{i} \sum_{j=0}^{k-\lceil\alpha\rceil} \Theta_{i, j, k} T_{j}^{*}(t)
$$

where

$$
\begin{aligned}
\Theta_{i, j, k} & =\frac{(-1)^{i-k} 2 i(i+k-1) ! \Gamma\left(k-\alpha+\frac{1}{2}\right)}{h_{j} \Gamma\left(k+\frac{1}{2}\right)(i-k) ! \Gamma(k-\alpha-j+1) \Gamma(k+j-\alpha+1)}, \\
j & =0,1, \ldots
\end{aligned}
$$

\section{Theorem 4.}

The error $\left|E_{T}(m)\right|=\left|D^{\alpha} x(t)-D^{\alpha} x_{m}(t)\right|$ in approximating $D^{\alpha} x(t)$ by $D^{\alpha} x_{m}(t)$ is bounded by

$$
\left|E_{T}(m)\right| \leq\left|\sum_{i=m+1}^{\infty} c_{i}\left(\sum_{k=\lceil\alpha\rceil}^{i} \sum_{j=0}^{k-\lceil\alpha\rceil} \Theta_{i, j, k}\right)\right|
$$

Proof.A combination of Eqs.(8), (10) and (18) leads to

$$
\begin{aligned}
\left|E_{T}(m)\right| & =\left|D^{\alpha} x(t)-D^{\alpha} x_{m}(t)\right| \\
& =\left|\sum_{i=m+1}^{\infty} c_{i}\left(\sum_{k=\lceil\alpha\rceil}^{i} \sum_{j=0}^{k-\lceil\alpha\rceil} \Theta_{i, j, k} T_{j}^{*}(t)\right)\right|,
\end{aligned}
$$

but $\left|T_{j}^{*}(t)\right| \leq 1$, so, we can obtain

$$
\left|E_{T}(m)\right| \leq\left|\sum_{i=m+1}^{\infty} c_{i}\left(\sum_{k=\lceil\alpha\rceil}^{i} \sum_{j=0}^{k-\lceil\alpha\rceil} \Theta_{i, j, k}\right)\right|
$$

and subtracting the truncated series from the infinite series, bounding each term in the difference, and summing the bounds completes the proof of the theorem. 


\section{Procedure solution of the fractional SIRC model and influenza $A$}

Consider the fractional SIRC model and influenza A of the type given in Eq.(1). In order to use Chebyshev collocation method, we first approximate $S(t), I(t), R(t)$ and $C(t)$ as

$$
\begin{array}{ll}
S_{m}(t)=\sum_{i=0}^{m} a_{i} T_{i}^{*}(t), & I_{m}(t)=\sum_{i=0}^{m} b_{i} T_{i}^{*}(t), \\
R_{m}(t)=\sum_{i=0}^{m} c_{i} T_{i}^{*}(t), & C_{m}(t)=\sum_{i=0}^{m} d_{i} T_{i}^{*}(t) .
\end{array}
$$

From Eqs.(1) and Theorem 1 we have

$$
\begin{aligned}
& \sum_{i=\lceil\alpha\rceil}^{m} \sum_{k=\lceil\alpha\rceil}^{i} a_{i} w_{i, k}^{(\alpha)} t^{k-\alpha}=\mu\left(1-\sum_{i=0}^{m} a_{i} T_{i}^{*}(t)\right) \\
& -\beta\left(\sum_{i=0}^{m} a_{i} T_{i}^{*}(t)\right)\left(\sum_{i=0}^{m} b_{i} T_{i}^{*}(t)\right)+\gamma\left(\sum_{i=0}^{m} d_{i} T_{i}^{*}(t)\right), \\
& \sum_{i=\lceil\alpha\rceil}^{m} \sum_{k=\lceil\alpha\rceil}^{i} b_{i} w_{i, k}^{(\alpha)} t^{k-\alpha}=\beta\left(\sum_{i=0}^{m} a_{i} T_{i}^{*}(t)\right)\left(\sum_{i=0}^{m} b_{i} T_{i}^{*}(t)\right)+ \\
& \sigma \beta\left(\sum_{i=0}^{m} d_{i} T_{i}^{*}(t)\right)\left(\sum_{i=0}^{m} b_{i} T_{i}^{*}(t)\right)-(\mu+\theta)\left(\sum_{i=0}^{m} b_{i} T_{i}^{*}(t)\right), \\
& \sum_{i=\lceil\alpha\rceil k=\lceil\alpha\rceil}^{m} \sum_{i}^{i} w_{i, k}^{(\alpha)} t^{k-\alpha}=(1-\sigma) \beta\left(\sum_{i=0}^{m} d_{i} T_{i}^{*}(t)\right)\left(\sum_{i=0}^{m} b_{i} T_{i}^{*}(t)\right) \\
& +\theta\left(\sum_{i=0}^{m} b_{i} T_{i}^{*}(t)\right)-(\mu+\delta) \sum_{i=0}^{m} c_{i} T_{i}^{*}(t), \\
& \sum_{i=\lceil\alpha\rceil}^{m} \sum_{k=\lceil\alpha\rceil}^{i} d_{i} w_{i, k}^{(\alpha)} t^{k-\alpha}=\delta\left(\sum_{i=0}^{m} c_{i} T_{i}^{*}(t)\right) \\
& -\beta\left(\sum_{i=0}^{m} d_{i} T_{i}^{*}(t)\right)\left(\sum_{i=0}^{m} b_{i} T_{i}^{*}(t)\right)-(\mu+\gamma)\left(\sum_{i=0}^{m} d_{i} T_{i}^{*}(t)\right) .
\end{aligned}
$$

We now collocate Eqs.(21) at $(m+1-\lceil\alpha\rceil)$ points $t_{p}(p=0,1, \ldots, m+1-\lceil\alpha\rceil)$ as

$$
\begin{aligned}
& \sum_{i=\lceil\alpha\rceil k=\lceil\alpha\rceil}^{m} \sum_{i}^{i} w_{i, k}^{(\alpha)} t_{p}^{k-\alpha}=\mu\left(1-\sum_{i=0}^{m} a_{i} T_{i}^{*}\left(t_{p}\right)\right) \\
& -\beta\left(\sum_{i=0}^{m} a_{i} T_{i}^{*}\left(t_{p}\right)\right)\left(\sum_{i=0}^{m} b_{i} T_{i}^{*}\left(t_{p}\right)\right)+\gamma\left(\sum_{i=0}^{m} d_{i} T_{i}^{*}\left(t_{p}\right)\right), \\
& \sum_{i=\lceil\alpha\rceil k=\lceil\alpha\rceil}^{m} \sum_{i}^{i} w_{i, k}^{(\alpha)} t_{p}^{k-\alpha}=\beta\left(\sum_{i=0}^{m} a_{i} T_{i}^{*}\left(t_{p}\right)\right)\left(\sum_{i=0}^{m} b_{i} T_{i}^{*}\left(t_{p}\right)\right)+ \\
& \sigma \beta\left(\sum_{i=0}^{m} d_{i} T_{i}^{*}\left(t_{p}\right)\right)\left(\sum_{i=0}^{m} b_{i} T_{i}^{*}\left(t_{p}\right)\right)-(\mu+\theta)\left(\sum_{i=0}^{m} b_{i} T_{i}^{*}\left(t_{p}\right)\right), \\
& \sum_{i=\lceil\alpha\rceil k=\lceil\alpha\rceil}^{m} \sum_{i}^{i} w_{i, k}^{(\alpha)} t_{p}^{k-\alpha}=(1-\sigma) \beta\left(\sum_{i=0}^{m} d_{i} T_{i}^{*}\left(t_{p}\right)\right)\left(\sum_{i=0}^{m} b_{i} T_{i}^{*}\left(t_{p}\right)\right) \\
& +\theta\left(\sum_{i=0}^{m} b_{i} T_{i}^{*}\left(t_{p}\right)\right)-(\mu+\delta) \sum_{i=0}^{m} c_{i} T_{i}^{*}\left(t_{p}\right), \\
& \sum_{i=\lceil\alpha\rceil k=\lceil\alpha\rceil}^{m} \sum_{i}^{i} d_{i} w_{i, k}^{(\alpha)} t_{p}^{k-\alpha}=\delta\left(\sum_{i=0}^{m} c_{i} T_{i}^{*}\left(t_{p}\right)\right) \\
& -\beta\left(\sum_{i=0}^{m} d_{i} T_{i}^{*}\left(t_{p}\right)\right)\left(\sum_{i=0}^{m} b_{i} T_{i}^{*}\left(t_{p}\right)\right)-(\mu+\gamma)\left(\sum_{i=0}^{m} d_{i} T_{i}^{*}\left(t_{p}\right)\right) . \quad(22)
\end{aligned}
$$

For suitable collocation points we use roots of shifted Chebyshev polynomial $T_{m+1-\lceil\alpha\rceil}^{*}(t)$.

Also, by substituting Eq.(20) in the initial conditions (2) we can obtain $4\lceil\alpha\rceil$ equations as follows

$$
\begin{aligned}
& \sum_{i=0}^{m}(-1)^{i} a_{i}=s_{0}, \quad \sum_{i=0}^{m}(-1)^{i} b_{i}=i_{0}, \\
& \sum_{i=0}^{m}(-1)^{i} c_{i}=r_{0}, \quad \sum_{i=0}^{m}(-1)^{i} d_{i}=c_{0} .
\end{aligned}
$$

Equations (22), together with the equations of the initial conditions (23), give $(4 m+4)$ of nonlinear algebraic equations which can be solved using the Newton iteration method, for the unknowns $a_{i}, b_{i}, c_{i}$ and $d_{i}$ for $i=0,1, \ldots, m$.

\section{Numerical implementation}

In this section, we implement the suggested technique to solve the system SIRC (1) with the constants $\mu=0.02, \beta=100, \delta=$ $1, \gamma=0.5, \sigma=0.05, \theta=73$, and the initial conditions

$$
S(0)=0.8, \quad I(0)=0.1, \quad R(0)=0.05, \quad C(0)=0.05 .
$$

The approximate solutions of the proposed system are given in figures 1-6 at different values of $\alpha$. In figures 1-2 we compared the obtained approximate solution at $\alpha=1$ using the proposed method with the numerical solution using fourth-order RungeKutta method, respectively. Also, in the figures 3-6, we presented the behavior of the approximate solution $\left(S_{m}(t), I_{m}(t), R_{m}(t)\right.$, respectively, $\left.C_{m}(t)\right)$ at $\alpha=0.9$.

From figures 1 and 2, we can confirm that the approximate solution is in excellent agreement with the solution using fourth order Runge-Kutta. Also, from figures 3-6, we can conclude that the behavior of the approximate solution depends on the order of the fractional derivative.

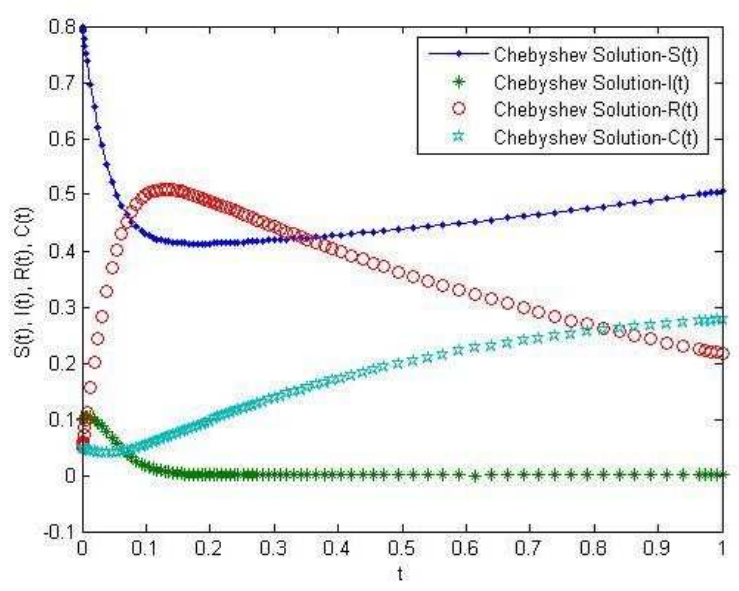

Figure 1. The behavior of the approximate solution using the proposed method at $\alpha=1$. 


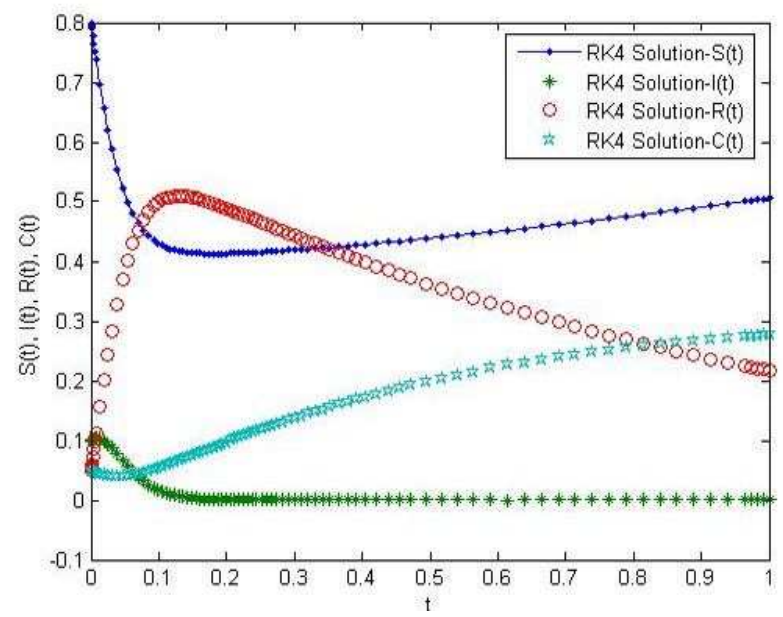

Figure 2. The numerical solution using Runge-Kutta method at $\alpha=1$.

\section{Conclusion and remarks}

In this article, the Chebyshev spectral method is implemented for solving the fractional SIRC model and influenza A. The fractional derivative is considered in the Caputo sense. The properties of the Chebyshev polynomials are used to reduce the proposed problem to the solution of a system of algebraic equations which is solved by using Newton iteration method. Special attention is given to study the convergence analysis and estimate an upper bound of the error of the derived formula. The obtained solutions using the suggested method are in excellent agreement with solutions from fourth-order Runge-Kutta and show that this approach can be solved the problem effectively. It is evident that the overall errors can be made smaller by adding new terms in the series (20). All computations in this paper are done using Matlab 8.

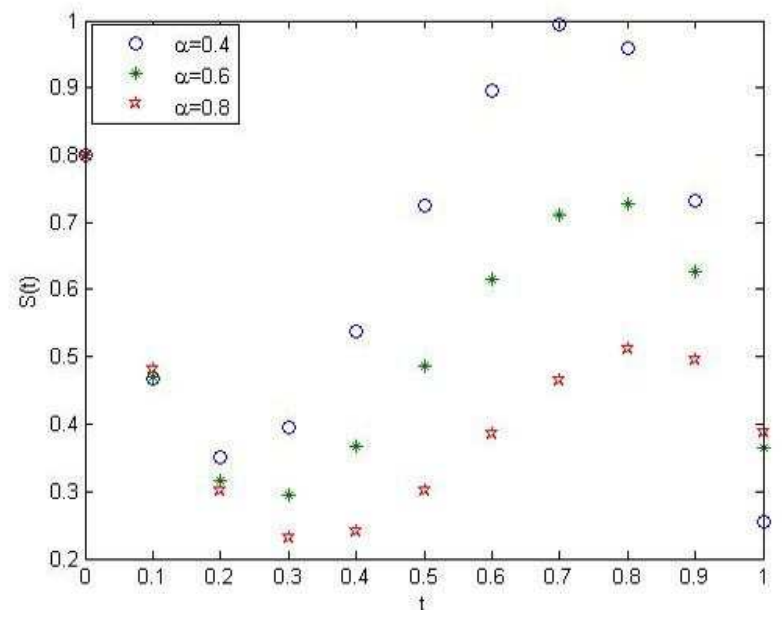

Figure 3. The behavior of the approximate solution $S(t)$.

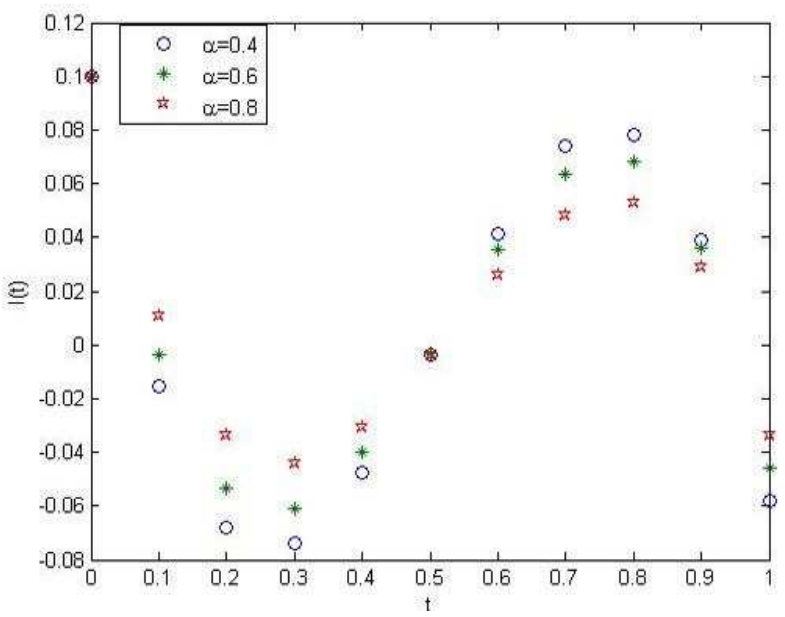

Figure 4. The behavior of the approximate solution $I(t)$.

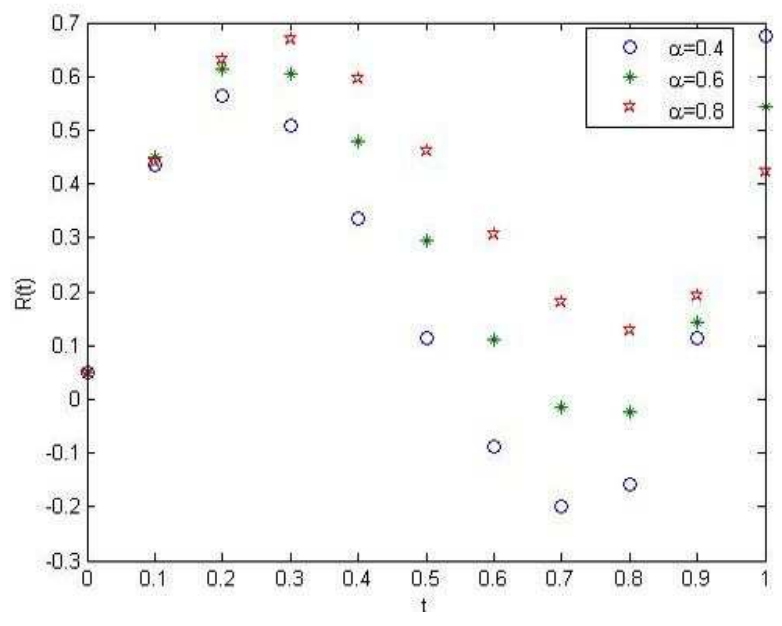

Figure 5. The behavior of the approximate solution $R(t)$.

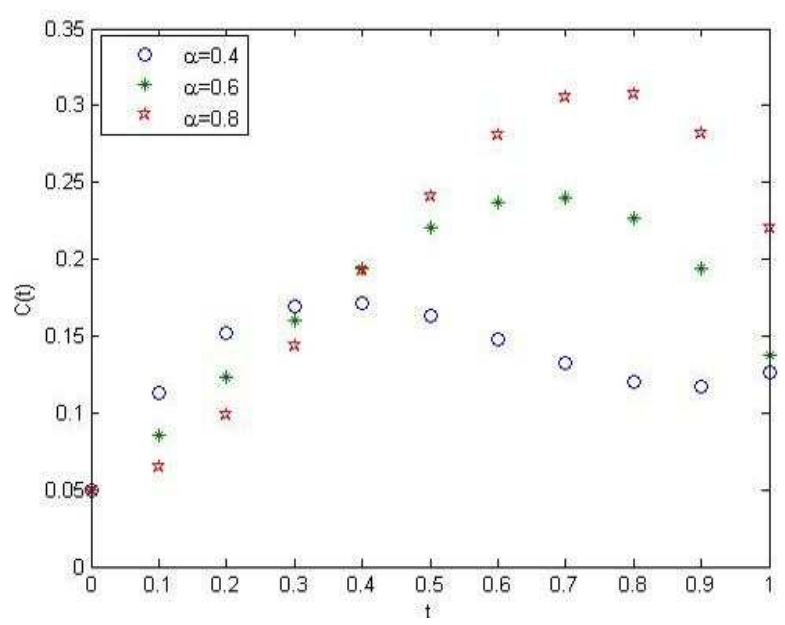

Figure 6. The behavior of the approximate solution $C(t)$. 


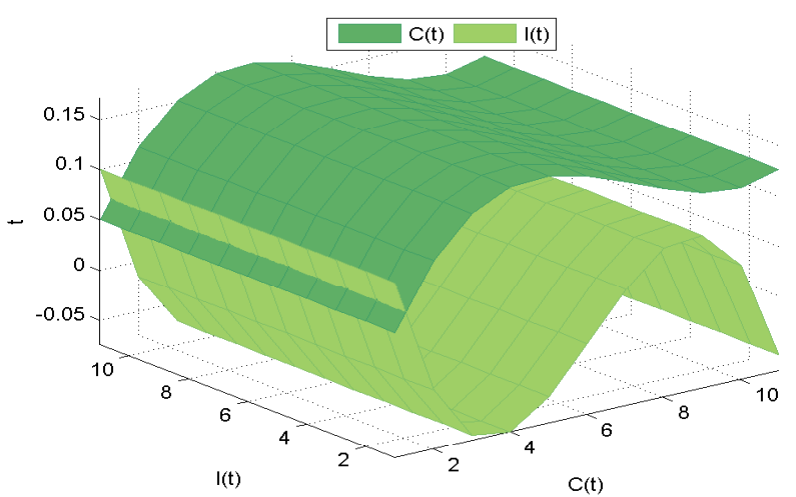

Figure 7.a. Relationship between $C(t)$ and $I(t)$.

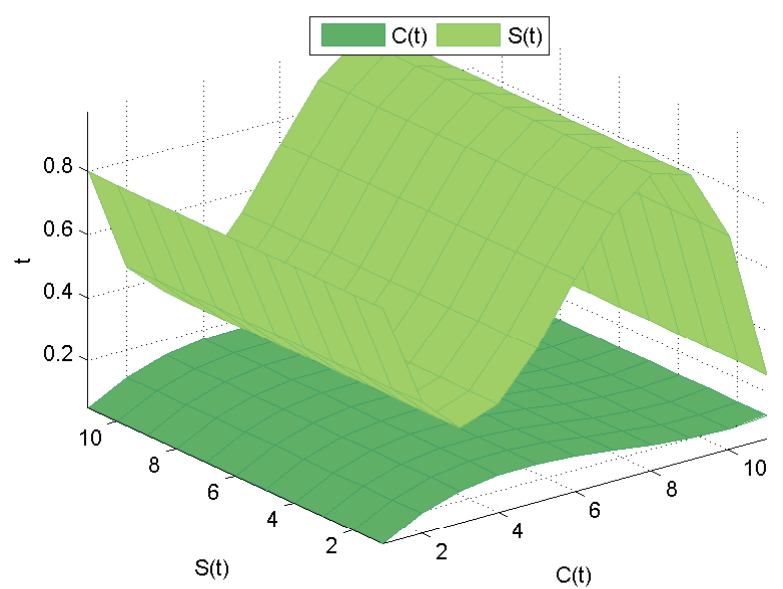

Figure 7.b. Relationship between $C(t)$ and $S(t)$.

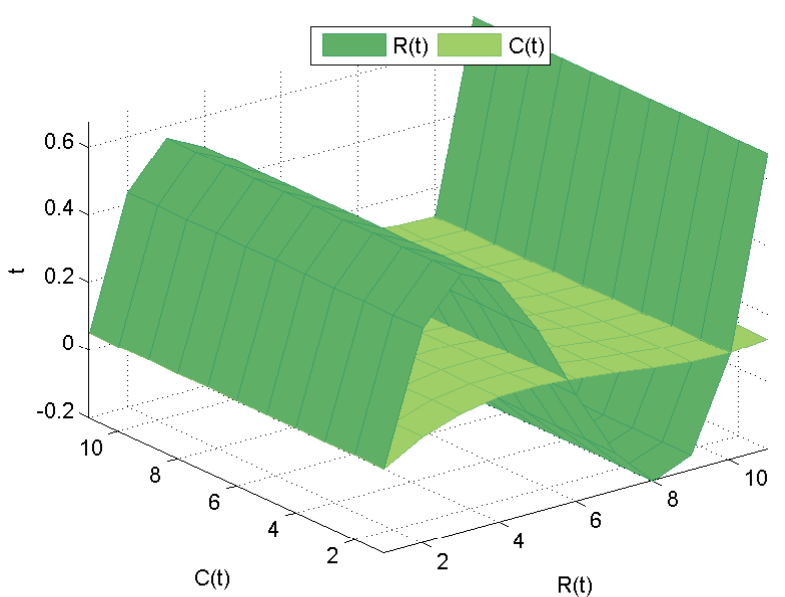

Figure 7.c. Relationship between $R(t)$ and $C(t)$.

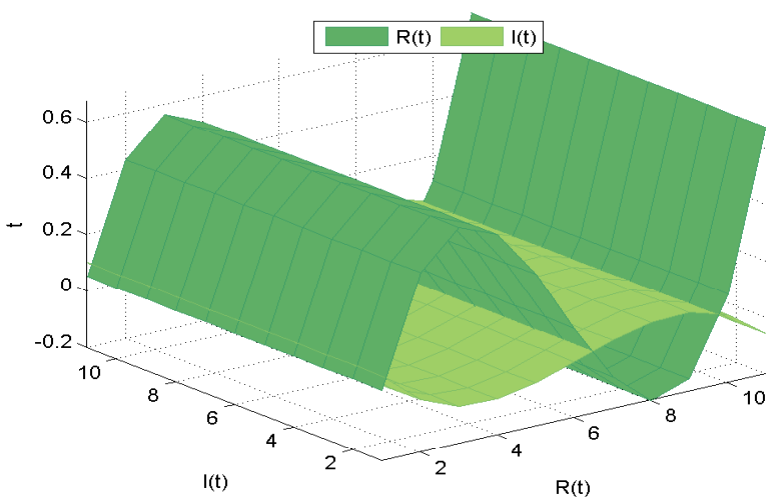

Figure 7.d. Relationship between $R(t)$ and $I(t)$.

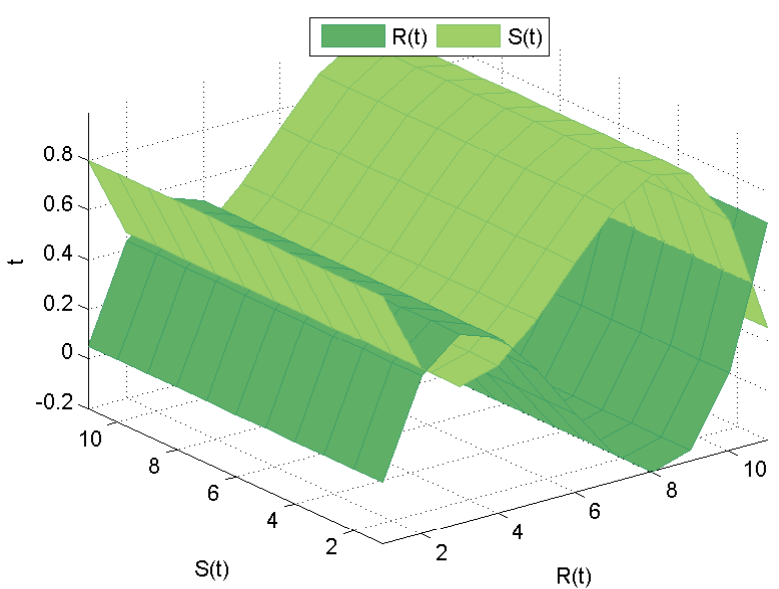

Figure 7.e Relationship between $R(t)$ and $S(t)$.

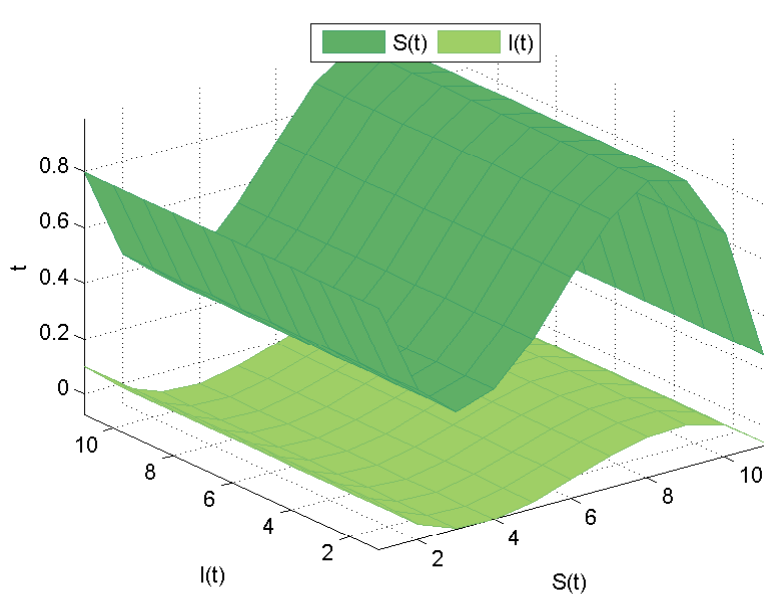

Figure 7.f. Relationship between $S(t)$ and $I(t)$. 
Previous figures 7(a-f) illustrate propagation of influenza $A$ virus along the time when $\alpha=0.4$ :

Figure (a) shows that cross-immune $C(t)$ intersects with infection at certain point $I(t)$ which means that an infection can be converted to cross-immune, also infection is capable of infected others, the cross immune is propagate slowly than infected figure which simulate the reality infection frequently changed.

Figure (b) shows no intersection between cross-immune $C(t)$ and suspectable $S(t)$ propagation which refer to suspectable can not easily converted to cross immune also suspectable is frequently changed unlike cross-immune since cross-immune expresses more advanced stage of the disease.

Figure (c) shows that intersections can occur more that once between recovered $R(t)$ and cross-immune $C(t)$ which reflects that a recovered state can be changed to cross-immune and this could happen frequently along the time, also cross-immune is very slow and steady in its changes along time unlike recovered which is very frequently changed since cross-immune is more advanced state than recovered.

Figure (d) shows that it is also possible for infected cases $I(t)$ to be converted to recovered cases $R(t)$ and vice vera several times which simulate the real world in which the status of disease can be switched between recover and infected, the rate of suspectable is changed more frequently than rate of infected, also rate of recovered is higher than rate of infection which is a good indication.

Figure (e) shows several intersections between recovered $C(t)$ and suspectable cases $S(t)$ which simulate the reality also the suspectable rate is higher than the recovered rate, and the frequency of changes in both figures is almost the same.

Figure (f) shows no intersection between suspectable cases $S(t)$ and infected cases $I(t)$ this means that changes between states is impossible, which means that there is no meaning to indicate a patient either suspectable or infected, one decision must be taken, also infection rate reflect the propagation of virus which is slower than just predicting with uncertainly that the virus is exist or no.

\section{Acknowledgement}

The authors are grateful to the anonymous referees for a careful checking of the details and for helpful comments that improved this paper. Special thanks to Prof. Mahmoud Abdel-Aty for sponsorship and interest in the acceptance and publication of this paper, appearing in the sky light.

\section{References}

[1] R. Anderson and R. May, Infectious Disease of Humans, Dynamics and Control, Oxford University Press, Oxford, UK, (1995).

[2] R. Casagrandi, L. Bolzoni, S. A. Levin, and V. Andreasen, The SIRC model and influenza A, Mathematical Biosciences, 200, 152-169 (2006).

[3] W. Chinviriyasit, Numerical modeling of the transmission dynamics of influenza, The First International Symposium on Optimization and Systems Biology, Beijing, China, 52-59 (2007).
[4] E. H. Elbasha, C. N. Podder and A. B. Gumel, Analyzing the dynamics of an SIRS vaccination model with waning natural and vaccine-induced immunity, Nonlinear Analysis, 12, 2692-2705 (2011).

[5] M. El-Shahed and A. Alsaedi, The fractional SIRC model and influenza A, Mathematical Problems in Engineering, 2011, 1-9 (2011).

[6] L. Jódar, R. J. Villanueva, A. J. Arenas, and G. C. González, Nonstandard numerical methods for a mathematical model for influenza disease, Mathematics and Computers in Simulation, 79, 622-633 (2008).

[7] D. W. Jordan and P. Smith, Nonlinear Ordinary Differential Equations, third ed., Oxford University Press, (1999).

[8] W. O. Kermack and A. G. McKendrick, Contributions to the mathematical theory of epidemics, Proceedings of Royal Society of London, 115, 700-721 (1927).

[9] M. M. Khader, On the numerical solutions for the fractional diffusion equation, Communications in Nonlinear Science and Numerical Simulation, 16, 2535-2542 (2011).

[10] M. M. Khader, Numerical treatment for solving the perturbed fractional PDEs using hybrid techniques, Journal of Computational Physics, 250, 565-573 (2013).

[11] M. M. Khader, Introducing an efficient modification of the VIM by using Chebyshev polynomials, Application and Applied Mathematics: An International Journal, 7, 283-299 (2012).

[12] M. M. Khader, Numerical treatment for solving fractional Riccati differential equation, Journal of the Egyptian Mathematical Society, 21, 32-37 (2013).

[13] M. M. Khader and A. S. Hendy, The approximate and exact solutions of the fractional-order delay differential equations using Legendre pseudospectral method, International Journal of Pure and Applied Mathematics, 74, 287-297 (2012).

[14] M. M. Khader and A. S. Hendy, A numerical technique for solving fractional variational problems, Mathematics Methods in Applied Sciences, 36, 1281-1289 (2013).

[15] M. M. Khader, N. H. Sweilam and A. M. S. Mahdy, An efficient numerical method for solving the fractional diffusion equation, J. of Applied Mathematics and Bioinformatics, 1, 112 (2011).

[16] M. M. Khader, N. H. Sweilam and A. M. S. Mahdy, Numerical study for the fractional differential equations generated by optimization problem using Chebyshev collocation method and FDM, Applied Mathematics and Information Science, 7, 2011-2018 (2013).

[17] M. M. Khader, T. S. EL Danaf and A. S. Hendy, A computational matrix method for solving systems of high order fractional differential equations, Applied Mathematical Modelling, 37, 4035-4050 (2013).

[18] K. B. Oldham and J. Spanier, The Fractional Calculus, Academic Press, New York, (1974).

[19] P. Palese and J. F. Young, Variation of influenza $A, B$, and $C$ viruses, Science, 215, 1468-1474 (1982).

[20] I. Podlubny, Fractional Differential Equations, Academic Press, New York, (1999).

[21] G. P. Samanta, Global dynamics of a nonautonomous SIRC model for influenza A with distributed time delay, Differential Equations and Dynamical Systems, 18, 341-362 (2010).

[22] M. A. Snyder, Chebyshev Methods in Numerical Approximation, Prentice-Hall, Inc. Englewood Cliffs, N. J. (1966). 
[23] N. H. Sweilam and M. M. Khader, A Chebyshev pseudospectral method for solving fractional integro-differential equations, ANZIAM, 51, 464-475 (2010).

[24] N. H. Sweilam, M. M. Khader and A. M. Nagy, Numerical solution of two-sided space-fractional wave equation using finite difference method, J. of Computional and Applied Mathematics, 235, 2832-2841 (2011).

[25] N. H. Sweilam, M. M. Khader and M. Adel, On the stability analysis of weighted average finite difference methods for fractional wave equation, Fractional Differential Calculus, 2, 17-29 (2012).

[26] N. H. Sweilam, M. M. Khader and A. M. S. Mahdy, Crank-Nicolson finite difference method for solving timefractional diffusion equation, Journal of Fractional Calculus and Applications, 2, 1-9 (2012).

[27] N. H. Sweilam, M. M. Khader and A. M. S. Mahdy, Numerical studies for solving fractional-order Logistic equation, Int. J. of Pure and Applied Mathematics, 78, 11991210 (2012).

[28] N. H. Sweilam, M. M. Khader and A. M. S. Mahdy, Numerical studies for fractional-order Logistic differential equation with two different delays, Journal of Applied Mathematics, 2012, Article ID 764894, 14 pages.

[29] N. H. Sweilam, M. M. Khader and W. Y. Kota, Numerical and analytical study for fourth-order integro-differential equations using a pseudo-spectral method, Mathematical Problems in Engineering, 2013, Article ID 434753, 7 pages.

[30] R. G. Webster, W. J. Bean, O. T. Gorman, T. M. Chambers and Y. Kawaoka, Evolution and ecology of influenza A viruses, Microbiological Reviews, 56, 152-179 (1992).

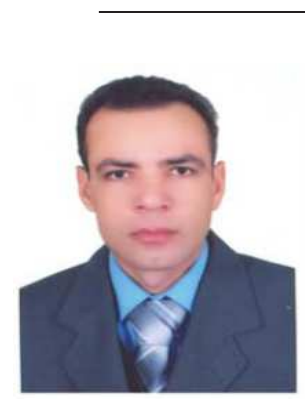

Mohamed M. Khader received the $\mathrm{PhD}$ degree in Mathematics Department Faculty of Science-Benha University-Egypt (2009). His research interests are in the areas of Pure Mathematics including the mathematical methods and numerical techniques for solving FDEs. He has published research articles in reputed international journals of mathematical. He is a referee of mathematical journals.

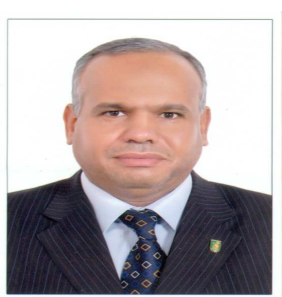

Nasser H. Sweilam analysis at the Department of Mathematics, Faculty of Science, Cairo University. $\mathrm{He}$ was a channel system Ph.D. student between Cairo University, Egypt, and TU-Munich, Germany. He received his Ph.D. in Optimal Control of Variational Inequalities, the Dam Problem. He is the Head of the Department of Mathematics, Faculty of Science, Cairo University, since May 2012. He is referee and editor of several international journals, in the frame of pure and applied Mathematics. His main research interests are numerical analysis, optimal control of differential equations, fractional and variable order calculus, bio-informatics and cluster computing and ill-posed problems.

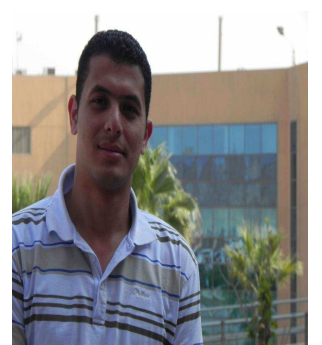

Amr M. S. Mahdy received the $\mathrm{PhD}$ degree in Mathematics Department-Faculty of Science, Zagazig University (2013). His research interests are in the areas of Pure Mathematics including the mathematical methods and numerical techniques for solving fractional differential equations. $\mathrm{He}$ has published research articles in reputed international journals of mathematical.

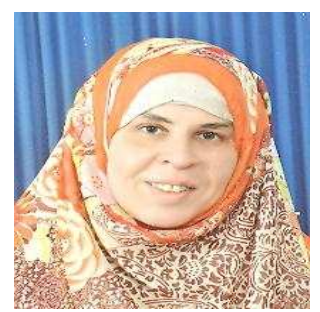

Nermeen K. A. Elmoniem $\mathrm{PhD}$ student in Mathematics Department-Faculty of Science, Cairo University. Her research interests are in the areas of Mathematical modeling and optimization and how to benefit from the huge amount of databases all over the world in mathematical modeling and

optimization. 